CBIE-LACLO 2015

Anais dos Workshops do IV Congresso Brasileiro de Informática na Educação (CBIE 2015)

\title{
Uma avaliação do Modelo Sala de Aula Invertida no Ensino Superior
}

\author{
Adriana D. Dantas ${ }^{1,2}$, Luiz S. O. Barbosa ${ }^{3}$, Isomar L. da Silva ${ }^{1}$, Thaís H. C. de \\ Castro ${ }^{1}$, Nara Vieira da Silva ${ }^{2}$, António R. da C. Neto ${ }^{2}$ \\ ${ }^{1}$ Instituto de Computação - Universidade Federal do Amazonas - (UFAM) \\ Av. Gen. Rodrigo Otávio, 6200, Setor Norte do Campus Universitário - Coroado I, CEP \\ 69077-000 -Manaus -AM -Brasil. \\ ${ }^{2}$ Instituto Federal de Educação Ciência e Tecnologia do Amazonas - (IFAM) \\ Rua Ferreira Pena, 1109 - Centro, CEP 69025-010 -Manaus -AM -Brasil. \\ ${ }^{3}$ Centro de Estudos Superiores de Itacoatiara- Cesit - Universidade do Estado do \\ Amazonas - (UEA) \\ Av. Mário Andreazza s/n - São Francisco CEP 690101-416 -Itacoatiara -AM -Brasil. \\ \{adriana.dantas, antonioneto\} @ifam.edu.br, luizsergio@gmail.com, \\ isomar.lima@msn.com, naraosga@gmail.com, thais@icomp.ufam.edu.br
}

\begin{abstract}
This article presents a case study that aimed to encourage active learning and behavior analysis of a graduate class in IT in the Federal Institute of Amazonas as the use of flipped classroom model. For the research, a case study was done during the offering of a discipline from the course. The result of this work was relevant to the whole team, to contribute in the capacity of teachers in changing the mentoring methodology, the students' attitude towards teaching and learning method in Distance Education.
\end{abstract}

Resumo. Este artigo apresenta uma avaliação que teve como objetivo incentivar a aprendizagem ativa e analisar o comportamento de uma turma de pós-graduação em Informática na Educação do Instituto Federal do Amazonas quanto à utilização do modelo sala de aula invertida. Para a realização da pesquisa foi feita uma avaliação durante a oferta de uma das disciplinas do curso. O resultado deste trabalho foi relevante para toda a equipe, no sentido de contribuir na preparação de professores, na mudança da metodologia de tutoria, na postura dos alunos em relação ao ensino e aprendizagem em Educação à Distância.

\section{Introdução}

A modalidade de Educação à Distância vem se tornando cada vez mais frequente nas instituições de ensino, assumindo um importante papel no cenário educacional do país, em particular na formação em nível superior de setores da população que dificilmente alcançariam essa categoria de escolaridade, por fatores sociais e econômicos diversos. Essa modalidade viabiliza a formação em horários flexíveis distintos dos horários em ambientes escolares presenciais, possui um alcance geográfico muito abrangente, assim como a possibilidade de redução de custos na formação profissional do cidadão [Ramos et al. 2014a]. 
No entanto, Valente (2013) afirma que o ensino superior nessa modalidade enfrenta atualmente dois grandes desafios. $O$ primeiro deles é em relação às salas de aula, cada vez mais vazias ou quando o aluno está presente, ele está fazendo outra coisa diferente do que acompanhar a aula. No trabalho de Ribeiro et al. (2014) é relatado bem a deficiência apresentada acima, segundo um dos desafios para superar os alarmantes índices de evasão na EaD passa pela identificação e compreensão das diferentes ações de efetividade que os gestores são chamados a intervir, pois estes através do seu poder de decisão: definem, organizam e implementam as ações necessárias para o sucesso do processo de ensino-aprendizagem. Sendo a evasão um aspecto que envolve o ensino à distância cada vez mais presente nas Instituições de Ensino Superior (IES) Ribeiro et. al (2014), caput Daudt e Behar, (2013), esta pesquisa busca identificar parâmetros que possam contribuir para minimizar os índices de desistência através de análises dos relatórios de conclusão e da correlação destes com a avaliação da percepção dos alunos ao final de duas edições do curso.

O segundo desafio apontado por Valente (2013) é a incapacidade de atender a grande demanda de alunos que querem ingressar no ensino superior. Assim, o modelo de universidade que faz pesquisa, gera conhecimento e distribui este conhecimento para poucos não se sustenta mais. No intuito de minimizar essa problemática, Valente (2013) e Herreid (2013) propõem a utilização de inovações metodológicas e ambos afirmam ainda que as soluções a serem adotadas exigem mudanças no processo de ensino e aprendizagem, que são muito mais profundas, especificamente com relação à sala de aula, ela terá de ser repensada na sua estrutura, bem como na abordagem pedagógica que tem sido utilizada.

Diante do exposto, o objetivo deste artigo é apresentar uma avaliação utilizando o modelo de sala de aula invertida, essa nova metodologia é uma tendência inovadora na modalidade de ensino à distância no país, com o intuito de incentivar a aprendizagem ativa e que demonstrou resultados relevantes não só para os alunos, mas para toda equipe do curso de pós-graduação.

\section{Referencial Teórico}

A Sala de Aula Invertida (SAI), em língua inglesa Flipped Classroom, é um modelo de rotação na qual os alunos estudam os conteúdos de modo on-line, em casa, na escola ou em algum outro espaço escolhido pelo aluno, e o tempo em sala de aula é reservado para atividades de aprendizagem com o acompanhamento do professor, relata [Mendonça, 2015].

A sala de aula invertida ou modelo instrutivo, foi desenvolvido por Jonathan Bergmann e Sams Aaron, em 2007 no intuito de fornecer instruções para alunos do ensino secundário que estavam faltando às aulas presencias de química, portanto, ausentes das instruções do professor.

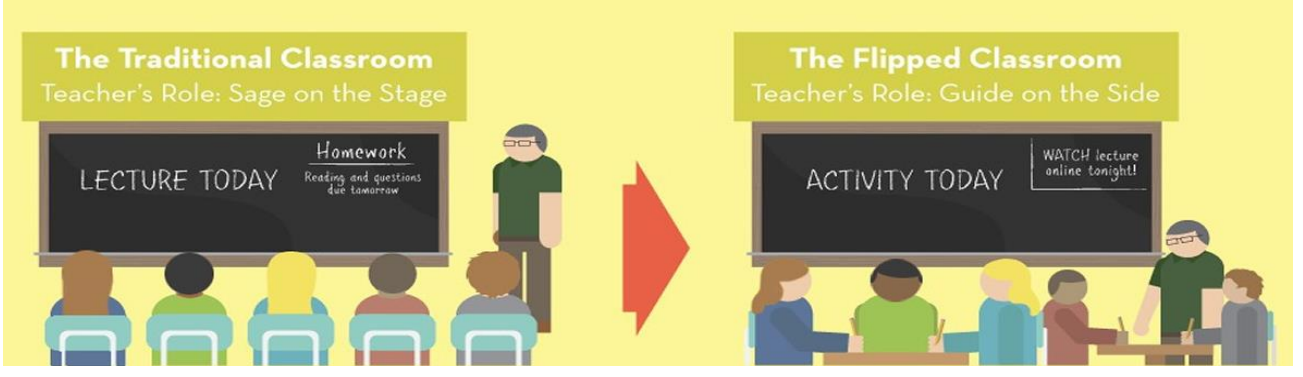

Figura 1. Método da Sala de Aula Invertida por Teaching \& Assessment, (2015) 


\section{CBIE-LACLO 2015}

Anais dos Workshops do IV Congresso Brasileiro de Informática na Educação (CBIE 2015)

De acordo com Mendonça (2015) no estudo on-line os alunos se apropriam do conteúdo de forma autônoma, utilizando-se de vídeo-aulas, textos, listas de exercícios e outros recursos indicados pelo professor por meio de um roteiro de aprendizagem previamente planejado, podendo ainda ampliar esse repertório a partir de suas pesquisas pessoais. Ao chegar à sala de aula os alunos já tinham todo o conhecimento referente ao conteúdo on-line, passando a se dedicar em tirar dúvidas sobre o material exposto, esclarecer resoluções de atividades, trabalhos em grupo e outras atividades voltadas ao conteúdo.

A sala de aula invertida está sendo vivenciada por diversas instituições em turmas que vão do jardim da infância até a universidade. No caso das escolas públicas, mais carentes de recursos, o que entra em cena é a iniciativa do professor, assim como sua formação continuada que o ajuda a ser mais conectado com essa prática pedagógica afirma [Lacoste 2014].

\section{Trabalhos Relacionados}

A partir de uma pesquisa teórica realizada, foram detectados poucos trabalhos referenciando o método da sala de aula invertida no Brasil, no entanto, em outros países foram encontrados vários exemplos de trabalhos referênciando o método há algum tempo.

A experiência descrita por Mendonça (2015), por exemplo, aconteceu na Escola Municipal Professora Thereza Favali Pocay em Salto Grande, Estado de São Paulo, nas disciplinas de História, Ciências e Geografia, com 18 alunos do $5^{\circ}$ ano do Ensino Fundamental, divididos em 9 duplas. Os momentos em sala de aula aconteceram em 2 aulas de 55 minutos. O objetivo da aplicação do método era que os alunos aprendessem sobre o ambiente em que viviam.

$\mathrm{Na}$ prática vivida por Tuker (2012) relata sobre a experiência vivida pelos autores do método, ambos perceberam que deveriam repensar radicalmente sobre a forma de como eles usariam o tempo de aula, a idéia central era de inverter a instrução comum da abordagem: com vídeos criados pelos professores e aulas interativas, instrução que costumavam ocorrer na classe seria acessada em casa, quando e quantas vezes quisessem. Dessa forma a sala de aula torna-se o lugar para se trabalhar através de problemas, avançar conceitos, e se envolver aprendendo em colaboração.

A experiência com a adoção do modelo SAI é a da professora Haberman que em seu blog My Flipped Classrom Experience, documenta seu projeto de pesquisa-ação NASA Endeavor Program sobre a Sala de Aula Invertida. A experiência aconteceu na Scottsbluff High School de Nebraska, com 129 alunos do $10^{\circ}$ ao $12^{\circ}$ ano, onde no Brasil é comparado ao $1^{\circ}, 2^{\circ}$ e $3^{\circ}$ ano do Ensino Médio, na disciplina de Biologia. O objetivo da aplicação era que os alunos aprendessem mais sobre a disciplina de forma consistente utilizando todo o conteúdo da sala de aula, afirma [Mendonça 2015].

O trabalho de Bishop (2013) fornece uma pesquisa abrangente de investigação prévia e contínua da sala de aula invertida. Esses estudos são caracterizados em várias dimensões entre outros, estes incluem os tipos de estudos em sala de aula e fora dela, a média de atividades utilizadas para avaliar o estudo, e metodologias características de cada estudo. Os resultados desta pesquisa mostram que a maioria dos estudos realizados 
até a data explora as percepções dos alunos e usam desenhos de estudo em grupo único. Os relatórios de percepções de estudantes da sala de aula invertida são um pouco mistos em relação à satisfação, mas geralmente com resultados positivos.

E Zhang et. al (2013) descreve uma experiência positiva da sala de aula invertida em um curso de pós-graduação, o artigo fala ainda da pespectiva da aprendizagem a partir do desenvolvimento da metodologia de gameficação dentro da sala de aula invertida. As atividades expostas de forma diferenciada com textos objetivos para os alunos facilitam o aprendizado.

No trabalho de Zhong et. al (2013) ressalta a importância do desing instrucional no desenvolvimento da sala de aula invertida, o mesmo afirma ainda que é a parte mais importante para a intenção da aplicação do método. O modelo de instrução simplificada deve ser utilizado de forma organizada dentro da sala de aula invertida.

\section{Avaliação}

\subsection{Problema}

Através de relatórios feitos pelos tutores do curso de pós-graduação em informática na educação foi detectado que o acesso às salas e as atividades são tardios, afetando assim o cronograma inicial da disciplina e consequentemente o andamento do curso.

Em uma análise rápida foi percebido que os alunos esperavam os professores para explicação do conteúdo e realização das atividades nas aulas presenciais, caracterizando-se como uma turma de alunos passivos, sempre a espera do professor para despertar o processo de aprendizagem.

\subsection{Objetivo}

Incentivar aprendizagem ativa a partir da aplicação do método da sala de aula invertida e analisar o comportamento e desempenho dos alunos em relação à utilização do método, essa avaliação foi realizada a partir da análise de dados gerados pela elaboração de atividades realizadas pelos alunos no intuito de identificar o potencial de aprendizagem da turma em relação às outras disciplinas.

\subsection{Procedimentos Metodológicos}

Esta avaliação contou com dois tipos de pesquisas, a teórica e a descritiva, o principal objeto de análise foi o número de acessos dos alunos a partir da realização de atividades no ambiente.

\subsection{Polo de Pesquisa}

O ambiente Moodle utilizado no Instituto Federal do Amazonas possui outras instâncias, o curso de pós-graduação em Informática na Educação do Instituto é ofertado para 4 polos, incluindo capital e interior, a pesquisa descrita foi realizada em 1 dos 4 polos.

\subsection{Preparação do Ambiente}

Para a preparação do ambiente foi necessário um estudo adequado de leituras de artigos e análise de alguns modelos de salas voltadas à aplicação desse método. Segundo 
Lacoste (2014) as práticas de sala de aula invertida consistem em oferecer aos alunos textos, vídeos ou conteúdo adicional para estudo fora da sala de aula.

Foram selecionadas atividades individuais e em grupo acompanhadas de vídeos, textos e cronogramas com aulas dinâmicas e práticas para estimular a realização das atividades no ambiente. Além disso, a sala passou por uma avaliação que contou com a participação de uma equipe composta por duas pedagogas, um design instrucional, dois professores da área de informática e pela coordenação do curso.

\subsection{Cenário do Estudo de Caso}

A sala de aula proposta contava com quatro tópicos, descritos da seguinte forma:

1 Tópico - Mostra a apresentação do Professor, apresentação da disciplina, plano de ensino da disciplina e cronograma com instruções de como realizar as atividades e datas de entrega de cada tarefa da disciplina.

$2^{\circ}$ Tópico - Apresentam textos com conteúdos resumidos, vídeos que abordam e descrevem o texto para facilitar o entendimento dos alunos sobre o conteúdo. O tópico conta ainda com duas atividades que são o fórum e o questionário.

$3^{\text {o }}$ Tópico - Apresentam 3 atividades, uma delas é o fórum, a segunda atividade é o envio de arquivo único, e a terceira atividade proposta foi em grupo, para realização dessa atividade os alunos contaram com a ajuda de vídeos que descreviam algumas dicas de como resolver o problema proposto.

$4^{\mathbf{0}}$ Tópico - Apresentam 2 atividades, o envio de arquivo único e o fórum, a primeira atividade foi uma apresentação sobre o tema proposto pelo professor para cada equipe, essas apresentações foram feitas para o professor como nota final da disciplina.

\subsection{Tipos de Atividades do Cenário}

\subsubsection{Atividades Individuais}

Os alunos acessaram o ambiente e realizaram as atividades que estavam expostas para eles, sendo:

1. Responder a um questionário onde as respostas estavam em um texto disponibilizado na sala onde eles deveriam fazer o download do arquivo para responder as perguntas.

2. Fazer o upload do arquivo.

3. Respostas no fórum a respeito dos textos lidos.

\subsubsection{Atividades em Grupo}

Os alunos foram divididos em grupos obedecendo ao critério da ordem alfabética, sendo:

1. Resolver em grupo um problema proposto sobre acessibilidade na escola. Para cada grupo foi distribuído uma dica diferente no intuito de ajudá-los a resolver o problema. Para resolver o problema, os membros do grupo deveriam interagir usando o chat ou mensagens como meio de comunicação. 
CBIE-LACLO 2015

Anais dos Workshops do IV Congresso Brasileiro de Informática na Educação (CBIE 2015)

2. O conteúdo do arquivo único deve conter a apresentação dos alunos a respeito das boas práticas de cada tema de proposto.

\subsection{Perfis dos Participantes}

Os participantes foram alunos do Curso de Pós-graduação em Informática na Educação e ocorreu durante a disciplina Acessibilidade e Informática na Escola Inclusiva. A turma é formada por 30 alunos, com idades entre 28 e 59 anos, muitos alunos são professores do ensino fundamental e médio da rede pública de ensino do estado do Amazonas.

Todos os participantes assinaram um Termo de Consentimento Livre e Esclarecido que descrevia todo o processo de pesquisa, o objetivo e a importância da veracidade da resposta de cada participante para a pesquisa.

\subsection{Execução da Avaliação}

A avaliação foi realizada em março de 2015, e teve um período de 30 dias, o mesmo tempo que leva a oferta de uma disciplina regular no curso, o conteúdo foi disponibilizado para os participantes na sala virtual 20 (vinte) dias antes da aula presencial, os alunos tiveram vários dias para visualizar todo o conteúdo e tentar responder as atividades do ambiente sozinhos ou em grupo, sem nenhuma ajuda primária do professor, somente utilizando o material disponível em sala de aula.

O professor antes de ministrar as aulas presenciais, verificou as questões mais problemáticas e dúvidas mais pertinentes dos alunos, essas dúvidas são trabalhadas em sala de aula no intuito de dar um feedback mais objetivo aos alunos. Durante a aula, as discussões são intercaladas e destinadas a expor as dificuldades que os alunos encontram em realizar as atividades. Algumas dúvidas são respondidas via mensagem, no fórum ou chat, de modo que a classe e o professor possam acompanhar o nível de compreensão sobre os conceitos em discussão.

Para a atividade colaborativa os alunos foram divididos em 4 grupos de 6 pessoas, e um grupo de 4 pessoas, dando um total de 5 grupos, onde cada aluno ficaria responsável por apresentar um trabalho sobre um tipo de deficiência, os temas ficaram divididos da seguinte forma:

1-Pessoas com deficiência Física/Mobilidade

2-Pessoas com deficiência Visual

3-Pessoas com deficiência Auditiva

4-Pessoas com deficiências Multiplas/Comunicação

5-Acessibilidade na Web/Recursos de Tecnologias Assistivas para percepção e operação.

Para essa atividade cada grupo ficou responsável por pesquisar sobre um mesmo tema, dessa forma no final cada grupo apresentou sua contribuição de pesquisa relacionada ao tema proposto, com contribuições relevantes para a resolução de problemas do cotidiano desses usuários que merecem toda a nossa atenção como qualquer outro aluno. 
Para realização dessa atividade foi disponilizado vídeos que contavam depoimentos vividos por pessoas com diversas deficiências, no intuito de sensibilizar os alunos e despertar o senso crítico para contribuir de alguma forma para o bem-estar desses alunos em sala de aula.

\subsection{Coleta dos Dados}

A coleta de dados foi feita no próprio ambiente virtual a partir dos logs dos alunos, foi realizado um questionário dentro da sala de aula da disciplina onde os alunos responderam quanto a algumas questões pertinentes ao estudo de caso. Todos os alunos responderam o questionário, mas nem todos os alunos fizeram todas as atividades.

De acordo com Ramos et al. (2014b) o Moodle dispõe da ferramenta "Pesquisa" que pode ser facilmente configurada para realizar pesquisas e enquetes com diferentes tipos de questões. A ferramenta permite exportar os dados coletados em formato de planilha, facilitando análises mais aprofundadas através da utilização de ferramentas estatísticas e de mineração de dados.

\section{Resultados da Avaliação}

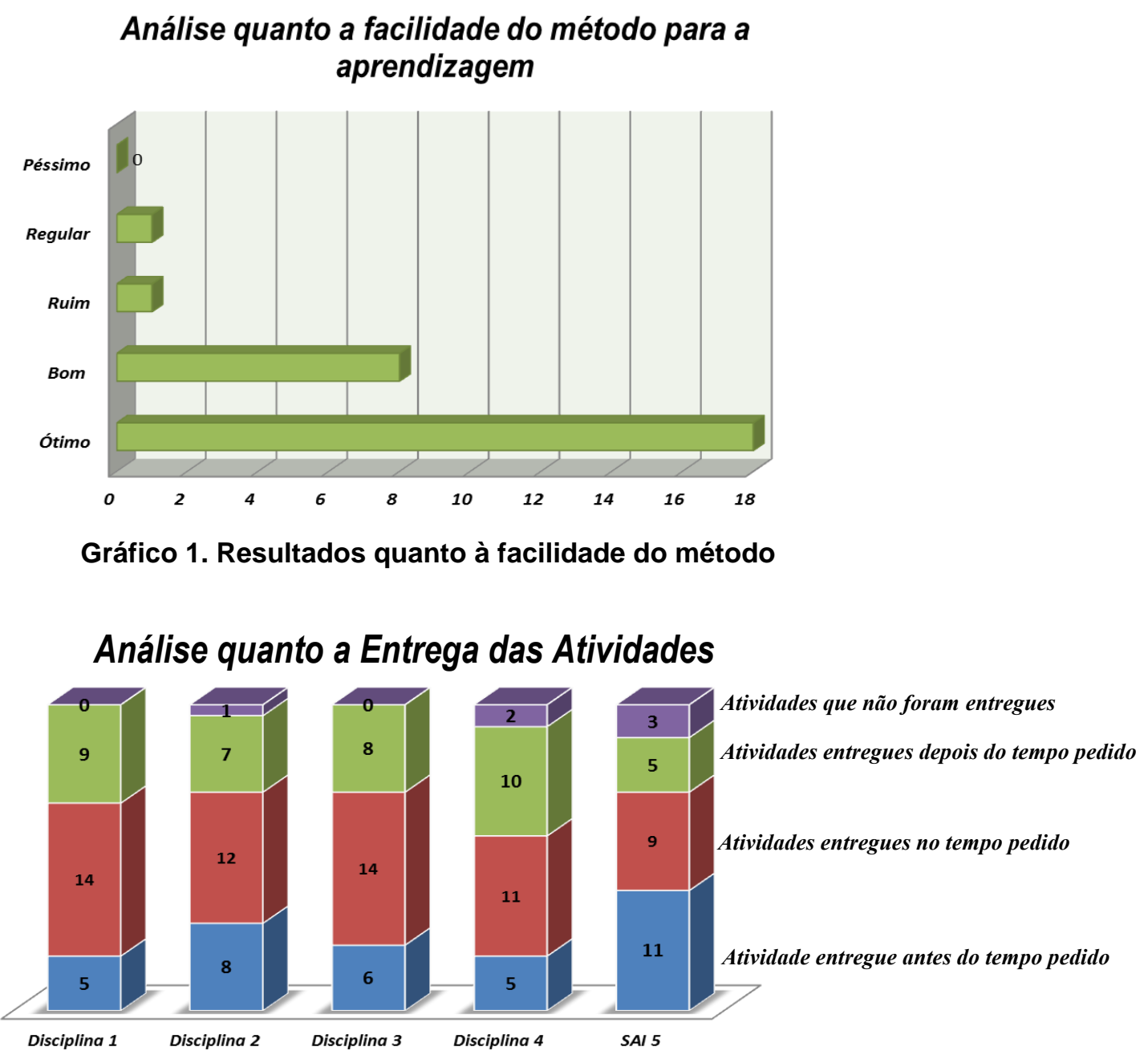

Gráfico 2. Resultados quanto a Entrega das Atividades 
Resultados sobre a Sala de Aula Invertida

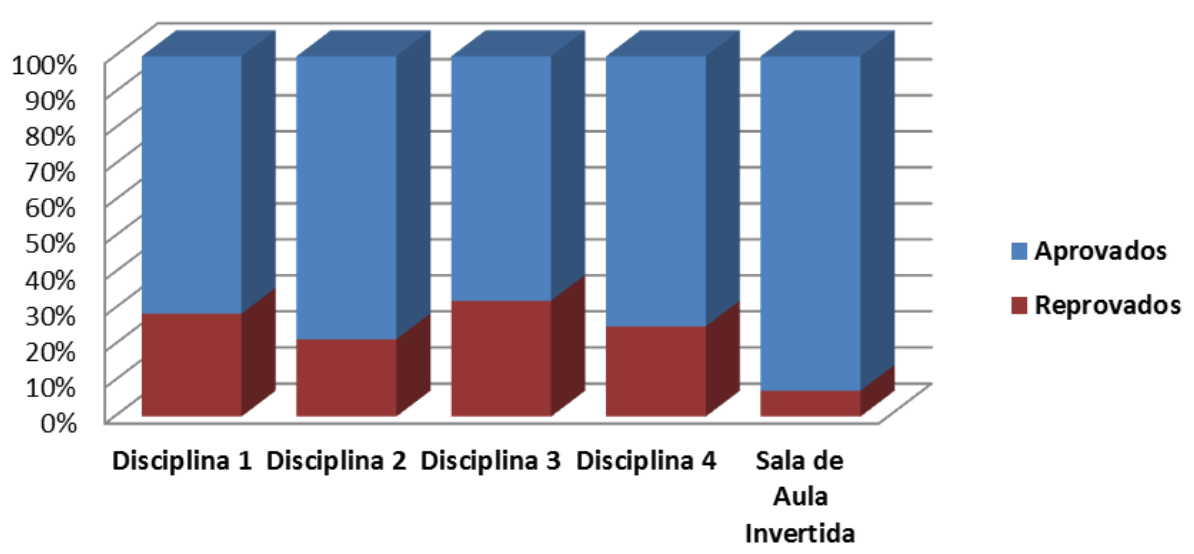

Gráfico 3. Resultados quanto à quantidade de alunos aprovados

No geral os resultados sobre a avaliação da sala de aula invertida mostraram-se positivos, em uma comparação feita entre a disciplina que utilizou a sala de aula invertida e as outras disciplinas que utilizaram o método convencional, foram notáveis as diferenças dos resultados. Os gráficos acima mostram os resultados em três aspectos diferentes e todos eles mostram resultados positivos na utilização do método da sala de aula invertida.

O gráfico 1 mostra que 18 de 28 alunos escolheram ótimo e 8 optaram por bom, quanto à facilidade de uso do método. No gráfico 2 mostra a entrega de atividades dos alunos em relação a todas as disciplinas e 11 de 28 alunos entregaram as atividades antes do tempo pedido, 9 alunos entregaram as atividades no tempo certo. No gráfico 3 mostra a quantidade de alunos aprovados e reprovados nas disciplinas em relação à utilização do método convencional e do método da sala de aula invertida.

\section{Conclusões do Estudo de Caso}

\subsection{Pontos de Vista Positivos do Método}

A partir da aplicação do método pôde ser percebida uma participação mais ativa dos alunos da turma, assim como o aumento no índice na realização das atividades, o que contribuiu para a aprovação de grande parte dos alunos na disciplina. A maioria dos alunos relatou que o modelo da sala de aula chamou a atenção deles e facilitou a realização das atividades.

A metodologia de tutoria também sofreu mudanças durante a oferta desta disciplina e se tornou naturalmente menos ativo devido à redução na quantidade de postagens de dúvidas dos alunos para o professor, dessa forma os tutores tiveram mais tempo para acompanhar melhor os alunos nas atividades em grupo. Para a coordenação do curso a qualidade da sala foi um dos pontos mais importantes e decisivos para que o método atingisse resultados relevantes para toda a equipe.

Valente (2013) afirma que na sala de aula invertida, professores qualificados são mais importantes do que nunca. São eles que devem definir o conteúdo, as instruções e traçar as estratégias de interação face a face. Durante a aula, devem observar e dar feedback, além de avaliar continuamente o trabalho do aluno. 
Foi identificada a necessidade de realizar treinamentos mais específicos com os professores do curso para as próximas disciplinas em relação à preparação de material mais elaborado para a sala de aula.

A preparação de um bom material e a forma como são organizados na sala naturalmente induz o aluno ao aprendizado imediato de forma precisa, sem a necessidade de leituras longas e cansativas.

\subsection{Pontos de Vista Negativos do Método}

A preparação da sala de aula deve ser minuciosa, o que leva um tempo e habilidade do professor na utilização das TCDS, a resistência de alguns alunos em realizar as atividades sem a instrução do professor, e o tempo de feedback do professor para os alunos que deve ser frequente.

De acordo com Valente (2013) como em qualquer modelo ou método de ensino, a sala de aula invertida pode ser adotada de forma errada. Logo, é importante notar que a iniciativa pode não funcionar para todos os professores e alunos, assim como para diferentes níveis e disciplinas. Nem todos os educadores terão sucesso e alguns alunos podem preferir as abordagens tradicionais, mas é uma boa alternativa para um mundo globalizado em que o uso da tecnologia e da comunicação aplicados à educação é cada vez maior.

\section{Trabalhos Futuros}

Como ilustrado ao longo deste trabalho, a investigação mais qualitativa e quantitativa precisa ser feita para identificar como o potencial do modelo pode ser maximizado. Mas a pesquisa existente demonstra claramente que o modelo de aprendizagem da sala de aula invertida pode ser uma maneira de criar um ambiente centrado no aluno.

Gorman (2012) observaram que qualquer educador que faz suas atividades centradas no aluno poderia proporcionar atividades na sala de aula que são baseados em ação, autênticidade, conectividade, colaboração, inovação de alto nível, envolvimento, experiência com base, aprendizagem aprofundada e auto realização.

Empregar estudos controlados com o objetivo de avaliar o desempenho dos alunos em um semestre também pode ser uma possibilidade de mostrar o potencial do método. Além disso, recomendamos que os pesquisadores utilizem e estimulem a sala de aula investida como forma de avaliar seu próprio material teórico e as atividades existentes na sala no intuito de melhorar seu design e forma de metodologia.

Espera-se que conforme as tecnologias e o acesso à internet de banda larga tornem-se mais acessíveis, ocorra um aumento da integração de tecnologias com os processos de aprendizagem e, consequentemente, cresça o interesse no desenvolvimento desse modelo específico de aprendizagem.

\section{Agradecimentos}

Esse trabalho recebeu apoio financeiro da Fundação de Amparo à Pesquisa do Estado do Amazonas-FAPEAM em parceria com a Universidade Federal do Amazonas-UFAM. Apoio pedagógico e suporte técnico do Instituto Federal do Amazonas-IFAM e da Universidade Estadual do Amazonas-UEA. Agradecemos a professora Amanda 


\section{CBIE-LACLO 2015}

Anais dos Workshops do IV Congresso Brasileiro de Informática na Educação (CBIE 2015)

Meincke Melo da Universidade Federal do Pampa pelas ideias das atividades da sala, aos alunos e tutores do Curso de Pós-Graduação em Informática na Educação que participaram das atividades propostas e aos avaliadores pela parceria e contribuição da pesquisa.

\section{Referências}

Bishop, J. L., Verleger, M.A., The Flipped Classroom: A Survey of the Research. $120^{\text {th }}$ ASEE Annual Conference \& Exposition - 2013 - June-Atlanta/USA.

Gorman, M. "Flipping the classroom... a goldmine of research and resources keep you on your feet." 21st Century Educational Technology (2012).

Herreid, C.F and Schiller, N.A. Case Studies and the Flipped Classroom. In Jornal of College Science Teaching - 2013-October - New York.

Lacorte, R.F. Práticas na Sala de Aula - Iberciência. Comunidad de Educadores para la Cultura Científica. 2014 - Deciembro - Toluca/México.

Mendonça, A.P. Tendência e Inovações no Ensino. Editora CRV-Curitiba - Abril de 2015.

Ramos, J. L. C., da Silva, R. F. P., Silva, J.C.S., Gomes, A. S. Adoção de Blended Learning: verificação do potencial de ampliação na Universidade Federal do Vale do São Francisco. Anais do XXV Simpósio Brasileiro de Informática na Educação (SBIE 2014a, Novembro - Dourados-MS).

Ramos, J. L. C., Rodrigues, R. L., Gomes, A. S., Silva, J. C. S. Analisando Fatores que Afetam o Desempenho de Estudantes Iniciantes em um Curso à Distância. Anais do XXV Simpósio Brasileiro de Informática na Educação (SBIE 2014b, Novembro Dourados-MS).

Ribeiro, G.O., Silva, T.E.V., Nunes,A.O., Pinto, F.A.P, Vasconcelos, F.H.L. Perspectivas para Reduação da Evasão em EaD a partir da Avaliação da Qualidade do Ensino Online. Anais do XXV Simpósio Brasileiro de Informática na Educação (SBIE 2014, Novembro - Dourados-MS).

Teaching \& Assessment-Flipping the Class for Active Learning - Disponível em: https://teaching.wikit.itu.dk/Flipped+Classroom. Acesso em: 12 de fevereiro de 2015.

Tucker, Bill. The Flipped Classroom-Online instruction at home frees class time for learning - Education Next/Winter 2012 -July-San Francisco/USA.

Valente, J.A. Aprendizagem Ativa no Ensino Superior: a proposta da sala de aula invertida.

Zhang Jinlei \& Zhang Baohui. Application of Game based Learning in Flipped Classroom. Journal of Distance Education, 2013-01

Zhong Xiaoliu, Song Shuqiang \& Jiao Lizhen. Instructional Design Based on the Idea of the Flipped Classroom in ICT Environment. Open Education Research, 2013-01 